\title{
Research Article \\ Quadratic Stabilization of LPV System by an LTI Controller Based on ILMI Algorithm
}

\author{
Wei Xie
}

Received 8 August 2006; Revised 23 February 2007; Accepted 25 May 2007

Recommended by José Manoel Balthazar

\begin{abstract}
A linear time-invariant (LTI) output feedback controller is designed for a linear parameter-varying (LPV) control system to achieve quadratic stability. The LPV system includes immeasurable dependent parameters that are assumed to vary in a polytopic space. To solve this control problem, a heuristic algorithm is proposed in the form of an iterative linear matrix inequality (ILMI) formulation. Furthermore, an effective method of setting an initial value of the ILMI algorithm is also proposed to increase the probability of getting an admissible solution for the controller design problem.
\end{abstract}

Copyright (c) 2007 Wei Xie. This is an open access article distributed under the Creative Commons Attribution License, which permits unrestricted use, distribution, and reproduction in any medium, provided the original work is properly cited.

\section{Introduction}

A linear parameter-varying (LPV) system is formalized as a certain type of nonlinear system, and is successfully applied in developing a control strategy which is based on classical gain-scheduled methodology [1]. Several tutorial papers and special publications concerning the gain-scheduled method of LPV control system are [2-7]. These gainscheduled LPV controller design approaches are applicable under the assumption that the dependent parameters can be measured online. In practical application, it is often difficult to satisfy this requirement. Therefore, it is crucial to design an effective LTI controller to get robust stability for an LPV plant with immeasurable dependent parameters. Here, these dependent parameters are assumed to vary in a polytopic space. In robust control framework of LPV system, a necessary and sufficient condition of quadratic stability for polytopic LPV system is formulated in terms of a finite LMIs optimization problem [8]. The underlying quadratic Lyapunov functions are also used to derive bounds on robust performance measures. Several heuristic procedures [9-13] have also been proposed to solve some control problems with nonconvex constraints such as a controller with fixed 
or reduced order of the decentralized structure. In [10], a method is presented to solve some controller design problems when structure constraints are imposed. The procedure is based on a two-stage optimization process, each stage requires the solution of a convex optimization problem based on a kind of LMI expression, in which either the controller gain matrix or the Lyapunov function is considered as the optimization variable.

This paper proposes a way of designing a quadratically stabilizing LTI output feedback controller for LPV system where dependent parameters vary in a polytopic space. Different from gain-scheduled LPV controller design, besides rank constraints, another constraint condition in which the controller matrix should be the same one for each vertex plant of LPV system is added. This problem still remains a complex issue and not numerically tractable. Here, a heuristic ILMI approach is presented to solve an admissible solution for this control problem. And a method of setting an initial value for the Lyapunov matrix is also proposed to increase the possibility of obtaining a feasible solution to the ILMI approach. The proposed method is better than random assignment of the initial value. Even though this approach is not guaranteed to converge globally, it may provide a useful alternative design tool in practice.

\section{Notation and definitions}

Consider an LPV plant $P(\theta(t))$ described by state space equations as

$$
\begin{aligned}
& \dot{x}(t)=A(\theta) x(t)+B_{u} u(t), \\
& y(t)=C_{y} u(t) .
\end{aligned}
$$

Here, state-space matrices have compatible dimensions of time-varying dependent parameters $\theta(t)=\left[\theta_{1}(t) \theta_{2}(t) \cdots \theta_{r}(t)\right]^{T} \in \mathbb{R}^{r}$. Moreover, we have the following assumptions.

(1) The system state matrix $A(\theta)$ is a continuous and bounded function and depends affinely on $\theta(t)$.

(2) The immeasurable real parameters $\theta(t)$ exist in the LPV plant and vary in a polytope $\Theta$ as

$$
\begin{aligned}
\theta(t) \in \Theta: & =\operatorname{Co}\left\{\omega_{1}, \omega_{2}, \ldots, \omega_{N}\right\} \\
& =\left\{\sum_{i=1}^{N} \alpha_{i}(t) \omega_{i}: \alpha_{i}(t) \geq 0, \sum_{i=1}^{N} \alpha_{i}(t)=1, N=2^{r}\right\} .
\end{aligned}
$$

(3) The LPV plant is quadratically detectable and quadratically stabilizable.

With the above assumptions, the system state matrix $A(\theta)$ can be expressed as

$$
A(\theta)=\sum_{i=1}^{N} \alpha_{i}(t) A_{i} \quad \text { with } \alpha_{i} \geq 0, \quad \sum_{i=1}^{N} \alpha_{i}=1 .
$$

Remark 2.1. It is assumed that the matrices $B_{u}, C_{y}$ of the LPV plant are time invariant. When they are time varying, a simple way is to satisfy the requirement by filtering the control input and output through lowpass filters. These filters should have sufficiently 
large bandwidth. Then, the dependent parameters are shifted into the state matrix $A(\theta)$ in [3].

Definition 2.2 (quadratic stability [14]). Considering a LPV system, $\dot{x}(t)=A(\theta) x(t)$ is said to be quadratically stable if and only if there exists $P>0$ such that

$$
A^{T}(\theta) P+P A(\theta)<0 .
$$

Remark 2.3. For polytopic LPV system, we have the equivalent conditions for (2.4) as

$$
A_{i}^{T} P+P A_{i}<0, \quad i=1, \ldots, N .
$$

It should be noted that if LPV system is quadratically stable one, it is also exponentially stable.

\section{Main results}

In this section, a LTI output feedback controller is designed to achieve quadratic stability for LPV system where dependent parameters vary in a polytopic space.

We seek to design a controller $\left(A_{K} \in \mathbb{R}^{n_{k} \times n_{k}}\right)$ of fixed order $n_{k}$ as

$$
\begin{gathered}
\dot{x}_{k}=A_{k} x_{k}+B_{k} y, \\
u=C_{k} x_{k}+D_{k} y,
\end{gathered}
$$

where $x_{K} \in \mathbb{R}^{n_{k}}$ is the controller state. Substituting (3.1) into (2.1), the closed-loop state matrix $A_{\mathrm{cl}}$ has the following expression:

$$
A_{\mathrm{cl}}(\theta)=\left[\begin{array}{cc}
A(\theta)+B_{u} D_{k} C_{y} & B_{u} C_{K} \\
B_{K} C_{y} & A_{K}
\end{array}\right] .
$$

First, the following definitions are made as

$$
J=\left[\begin{array}{cc}
A_{k} & B_{k} \\
C_{k} & D_{k}
\end{array}\right], \quad \bar{A}(\theta)=\left[\begin{array}{cc}
A(\theta) & 0 \\
0 & 0
\end{array}\right], \quad \bar{B}_{u}=\left[\begin{array}{cc}
0 & B_{u} \\
I & 0
\end{array}\right], \quad \bar{C}_{y}=\left[\begin{array}{cc}
0 & I \\
C_{y} & 0
\end{array}\right],
$$

which are totally dependent on the state-space matrices of the controller and the LPV plant. Then, the closed-loop relation is parameterized in terms of the controller realization as

$$
A_{\mathrm{cl}}(\theta)=\bar{A}(\theta)+\bar{B}_{u} J \bar{C}_{y}
$$

Theorem 3.1. Suppose LPV system is given in (3.4), and then the following are equivalent conditions.

(1) The closed-loop state matrix $A_{c l}(\theta)$ is quadratically stable.

(2) There exist a symmetric positive definite matrix $P$ and matrix $J$ such that

$$
\bar{A}(\theta) P+P \bar{A}^{T}(\theta)+\bar{B}_{u} J \bar{C}_{y} P+P \bar{C}_{y}^{T} J^{T} \bar{B}_{u}^{T}<\delta I
$$


4 Mathematical Problems in Engineering

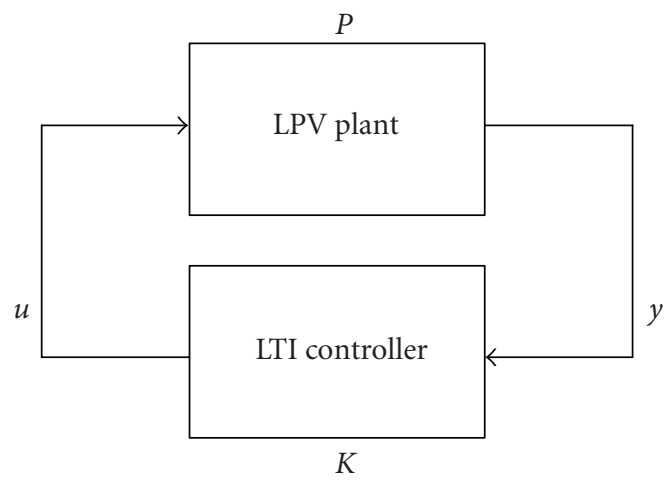

Figure 3.1. Relevant LPV control scheme.

or

$$
\bar{A}_{i} P+P \bar{A}_{i}^{T}+\bar{B}_{u} J \bar{C}_{y} P+P \bar{C}_{y}^{T} J^{T} \bar{B}_{u}^{T}<\delta I,
$$

$i=1, \ldots, N$, for $\delta$ being a negative scalar value.

Proof. According to Definition 2.2, the claims (3.5) or (3.6) can be established easily.

From (3.6), system matrix $J$ of the controller (3.1) should be the same one for each vertex plant of LPV system (3.2): it is also a nonconvex constraint and difficult to be solved. In the following section, necessary conditions for the existence of a constant matrix $J$ for (3.6) are presented, then a heuristic ILMI algorithm is presented to supply a solution of $J$ for (3.6). The choosing of an appropriate initial value to ILMI is very important to converge quickly to a feasible solution. Here, a method of setting an initial value to ILMI algorithm is also proposed.

THeOREM 3.2. Given an LPV plant (2.1), if there exists a fixed order LTI controller of order $n_{k}$ that makes the closed-loop LPV system as Figure 3.1 quadratically stable, then there exist $n \times n$ symmetric positive definite matrices $X, Y$ such that

$$
N_{o}^{T}\left(A^{T}(\theta) X+X A(\theta)\right) N_{o}<0, \quad N_{c}^{T}\left(Y A(\theta)+A^{T}(\theta) Y\right) N_{c}<0 .
$$

Using the polytopic characteristic of the LPV plant, (3.7) can be equivalent to

$$
\begin{gathered}
N_{o}^{T}\left(A_{i}^{T} X+X A_{i}\right) N_{o}<0, \quad N_{c}^{T}\left(Y A_{i}+A_{i}^{T} Y\right) N_{c}<0, \\
{\left[\begin{array}{cc}
X & I \\
I & Y
\end{array}\right] \geq 0,} \\
\operatorname{rank}\left[\begin{array}{cc}
X & I \\
I & Y
\end{array}\right] \leq n+n_{k},
\end{gathered}
$$

where $N_{o}$ and $N_{c}$ are full column rank matrices such that

$$
\operatorname{Im} N_{o}=\operatorname{ker} C_{y}, \quad \operatorname{Im} N_{c}=\operatorname{ker} B_{u}^{T} .
$$


The proof of the theorem can be easily taken from earlier results $[3,15]$.

Theorem 3.2 tells us necessary conditions of the existence of a stabilizing output feedback LTI controller for the LPV plant (2.1). Meanwhile, it also provides an efficient method for setting an initial value of the common Lyapunov matrix $P$, which is used to construct a stabilizing output feedback LTI controller.

Remark 3.3. Now, let us overview some results of LPV controller design for LPV plant. Consider the LPV plant (2.1), since this plant is assumed to be quadratically stabilizable and quadratically detectable, (3.8)-(3.9) are sufficient and necessary conditions for the existence of such a full-order gain-scheduled LPV controller that quadratically stabilizes LPV plant (2.1). In contrast to gain-scheduled LPV controller design [3], here only an LTI controller is designed to quadratically stabilize the LPV plant and conditions (3.8)-(3.9) become not sufficient but necessary just as Theorem 3.2.

Note that the matrix inequality (3.6) is a bilinear matrix problem with the constraint that controller gain matrix should be constant, and it is a nonconvex optimization problem. Here, a heuristic approach of alternately solving convex optimization problems is proposed based on LMI formulation. We minimize $\delta$, over $P$ and $J$, subject to (3.6). This problem is a convex optimization problem in $J$ and $\delta$ for fixed $P$, and is convex in $P$ and $\delta$ for fixed $J$. It also should be noted that this approach is guaranteed to converge, but not necessarily to the global optimum of the problem. The assignment of a proper initial value to $P$ is the key to enhance probability of converging to the global optimum. Here, conditions (3.8)-(3.9) supply necessary conditions for the existence of such an LTI controller of order $n_{k}$. Therefore, conditions (3.8)-(3.9) of Theorem 3.2 also give us an effective method of setting an initial value to $P$.

Therefore, the ILMI algorithm proceeds as shown in Algorithm 3.1.

If, after the procedure is alternated several times, solution $J$ is still infeasible, there are two cases: one is that a feasible $J$ may still exit, for this procedure does not necessarily guarantee to the solution $J$; the other is that the LPV plant may not be quadratically stabilizable by only an LTI controller.

\section{Numerical example}

In this section, two numerical examples are considered to illustrate the proposed method. All LMI-related computations are performed with LMI toolbox of Matlab [4].

Example 4.1. We consider the problem of controlling the yaw angles of a satellite system that appears in [4]. The satellite system consisting of two rigid bodies joined by a flexible link has the state-space representation as

$$
\left[\begin{array}{c}
\dot{\theta}_{1} \\
\dot{\theta}_{2} \\
\ddot{\theta}_{1} \\
\ddot{\theta}_{2}
\end{array}\right]=\left[\begin{array}{rrrr}
0 & 0 & 1 & 0 \\
0 & 0 & 0 & 1 \\
-k & k & -f & f \\
k & -k & f & -f
\end{array}\right]\left[\begin{array}{c}
\theta_{1} \\
\theta_{2} \\
\dot{\theta}_{1} \\
\dot{\theta}_{2}
\end{array}\right]+\left[\begin{array}{l}
0 \\
0 \\
0 \\
1
\end{array}\right] u, \quad y=\left[\begin{array}{llll}
1 & 0 & 0 & 0 \\
0 & 1 & 0 & 0 \\
0 & 0 & 1 & 0 \\
0 & 0 & 0 & 1
\end{array}\right]\left[\begin{array}{c}
\theta_{1} \\
\theta_{2} \\
\dot{\theta}_{1} \\
\dot{\theta}_{2}
\end{array}\right],
$$


Step 1.

Set initial value $i=0$, obtain $\quad P_{i}=\left[\begin{array}{cc}X & X_{2} \\ X_{2}^{T} & I\end{array}\right]$

subject to (3.8)-(3.9), where $X-Y^{-1}=X_{2} X_{2}^{T}$.

Let $\delta_{i}$ be an arbitrary large positive real number.

$\delta_{\text {old }}=\delta_{i}$.

Step 2.

Repeat \{

OP1: Solve eigenvalue problem, "minimize $\delta_{i 1}$, over $J_{i}$ and $\delta_{i 1}$, subject to (3.6);"

$\delta_{i}=\delta_{i 1 \mathrm{opt}}, J_{i}=J_{\mathrm{opt}}$.

If $\delta_{i}<0$, exit. $J_{i}$ is an admissible solution.

OP2: Solve eigenvalue problem, "minimize $\delta_{i 2}$, over $P_{i}$ and $\delta_{i 2}$, subject to (3.6)

and $P_{i}>0$ ";

$P_{i+1}=P_{i 2 \mathrm{opt}} . \delta_{i}=\delta_{i 2 \mathrm{opt}}$.

If $\delta_{i}<0$, exit. $J_{i}$ is an admissible solution.

If $\left\|\delta_{i}-\delta_{\text {old }}\right\|<\gamma$, a predetermined tolerance, exit.

Else $\delta_{\text {old }}=\delta_{i}$.

$i=i+1$.

\}

Algorithm 3.1

where $k$ and $f$ are torque constant and viscous damping, which vary in the following uncertainty ranges: $k \in\left[\begin{array}{ll}0.09 & 0.4\end{array}\right]$ and $f \in\left[\begin{array}{ll}0.0038 & 0.04\end{array}\right]$. A state-feedback controller $u=K x$ is designed to achieve quadratic stability for all possible parameter trajectories in the polytopic space. The pre-determined tolerance $\gamma$ is set to $1.0 e-4$. The following two cases are considered.

(1) Setting an arbitrary matrix to the initial $P$ such as identity matrix. After 12 iterations, $\delta_{12}$ converges to -0.0857 , therefore solution $K$ is found as

$$
K=\left[\begin{array}{llll}
1061463.3 & -1061463.3 & -258208.45 & -7338.2
\end{array}\right]
$$

(2) Setting an initial matrix to $P$ proposed in this paper. In this case, a state feedback is considered to construct, then an initial matrix of $P$ satisfying (3.8) is chosen as

$$
P_{0}=\left[\begin{array}{rrrc}
961.4 & 518.14 & -118.4 & 278.06 \\
518.1 & 930.3 & -247.3 & -167.8 \\
-118.4 & -247.3 & 95.46 & -55.25 \\
278.06 & -167.8 & -55.25 & 972.54
\end{array}\right] \text {. }
$$

Using the initial matrix $P_{0}$, after only 1 iteration, $\delta_{1}$ converges to -9395817.73 . An admissible $K$ is found as

$$
K=\left[\begin{array}{llll}
10541311.8 & -24814284.7 & -60435459.05 & -15945712.7
\end{array}\right]
$$

Therefore, the proposed method has a quicker convergence to a feasible solution than the method of setting an arbitrary matrix as the initial matrix $P$. 
Example 4.2. A classical example of parameter-varying unstable plant that can be viewed as a mass-spring-damper system with time-varying spring stiffness is considered [16]. The state-space equation of this unstable LPV plant is as follows:

$$
\begin{gathered}
A(\theta)=\left[\begin{array}{cc}
0 & 1 \\
-0.5-0.5 \theta & -0.2
\end{array}\right], \quad B_{u}=\left[\begin{array}{l}
0 \\
1
\end{array}\right], \\
C_{y}=\left[\begin{array}{ll}
1 & 0
\end{array}\right], \quad D_{u y}=0 .
\end{gathered}
$$

Here, the scope of time-varying parameter $\theta(t)$ is assumed in the polytope space $\Theta:=$ Co $\{-1,1\}$. An LTI output feedback controller is designed to achieve quadratic stability for all possible parameter trajectories in the polytopic space. The predetermined tolerance $\gamma$ is set to $1.0 e-4$.

Just like Example 4.1, the following two cases are considered.

(1) Setting an arbitrary matrix to the initial $P$, such as identity matrix. After 5 iterations, $\delta_{5}$ converges to 0.163 , which is larger than zero. Therefore solution $J$ is found infeasible.

(2) Setting an initial matrix to $P$ proposed in this paper. In this case, a full-order output feedback controller is considered to construct, then an initial matrix of $P$ satisfying (3.8)(3.9) is as follows:

$$
P_{0}=\left[\begin{array}{rrcc}
17.62 & -11.99 & 4.01 & -1.22 \\
-11.99 & 35.23 & -1.22 & 5.80 \\
4.01 & -1.22 & 1 & 0 \\
-1.22 & 5.80 & 0 & 1
\end{array}\right]
$$

Using the initial matrix $P_{0}$, after only 1 iteration, $\delta_{1}$ converges to -3.998 . An admissible $J$ is solved as

$$
J=1.0 e 8 *\left[\begin{array}{ccc}
-4.00 & -0.53 & -7.8 e-7 \\
-0.53 & -5.56 & 2.94 e-7 \\
3.76 e-8 & 6.0 e-10 & 2.0 e-7
\end{array}\right]
$$

Therefore, an LTI output feedback controller to satisfy quadratic stability of closedloop LPV system is constructed as

$$
\begin{gathered}
A_{k}=1.0 e 8 *\left[\begin{array}{ll}
-4.00 & -0.53 \\
-0.53 & -5.56
\end{array}\right], \quad B_{K}=\left[\begin{array}{l}
-78 \\
29.4
\end{array}\right], \\
C_{K}=\left[\begin{array}{ll}
3.76 & 0.06
\end{array}\right], \quad D_{k}=20 .
\end{gathered}
$$

When the trajectory of dependent parameter is assumed as $\theta(t)=0.63+0.1 \cdot e^{-t}$, the trajectory of the output of this plant can be drawn for the initial values $x(0)=\left[\begin{array}{ll}-0.25 & 0.15\end{array}\right]^{T}$ as shown in Figure 4.1.

Comparing these two cases above, numerical examples demonstrate that the proposed method of setting the initial value to ILMI algorithm is more efficient than the method of setting an arbitrary matrix as the initial value. 


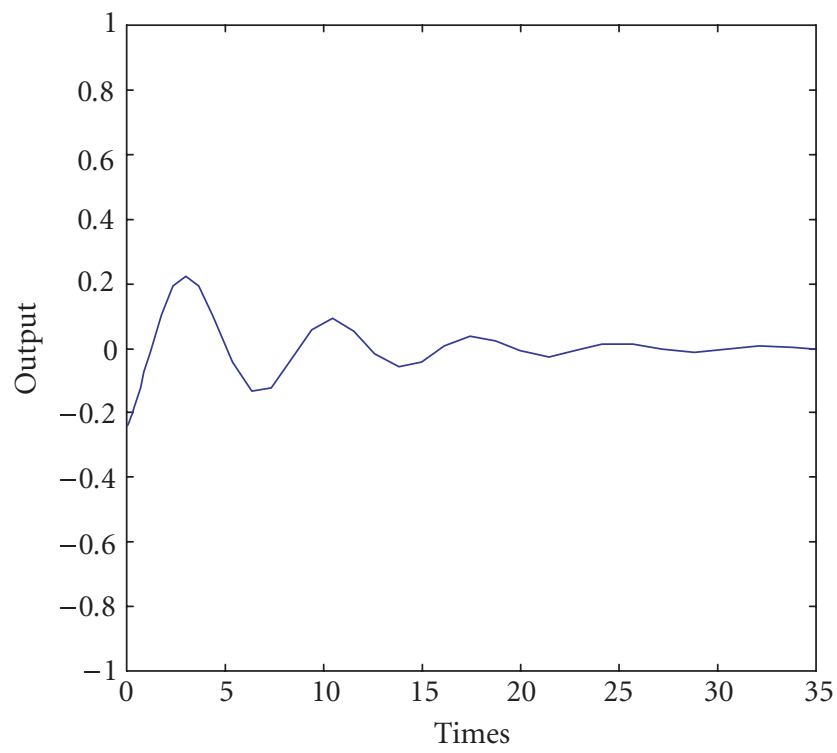

FIgURE 4.1. Trajectory of the output of this plant with initial values $x(0)=\left[\begin{array}{ll}-0.25 & 0.15\end{array}\right]^{T}$.

\section{Conclusions}

In this paper, an LTI output feedback controller has been designed for LPV system to ensure that the closed-loop system achieves quadratic stability for all possible dependent parameters in a polytopic space. A heuristic iterative algorithm to solve such a controller has been presented in terms of LMI formulation. It also should be noted that the procedure is heuristic and the choice of initial value is important to ensure convergence to an acceptable solution. Finally, some numerical examples have been presented to illustrate the design method.

\section{Acknowledgement}

This work is supported by the Guangdong Natural Science Foundation (Grant no. 07006470).

\section{References}

[1] J. S. Shamma and M. Athans, "Analysis of gain scheduled control for nonlinear plants," IEEE Transactions on Automatic Control, vol. 35, no. 8, pp. 898-907, 1990.

[2] P. Apkarian and P. Gahinet, "A convex characterization of gain-scheduled $\mathscr{H}_{\infty}$ controllers," IEEE Transactions on Automatic Control, vol. 40, no. 5, pp. 853-864, 1995.

[3] P. Apkarian, P. Gahinet, and G. Becker, "Self-scheduled $\mathscr{H}_{\infty}$ control of linear parameter-varying systems: a design example," Automatica, vol. 31, no. 9, pp. 1251-1261, 1995.

[4] P. Gahinet, A. Nemirovski, A. J. Laub, and M. Chilali, MATLAB LMI Control Toolbox, The MathWorks, Natick, Mass, USA, 1995.

[5] D. J. Leith and W. E. Leithead, "Survey of gain-scheduling analysis and design," International Journal of Control, vol. 73, no. 11, pp. 1001-1025, 2000. 
[6] W. Xie, "Quadratic L2 gain performance LPV system design by a LTI controller with ILMI algorithm," IEE Proceedings of Control Theory and Applications, vol. 152, no. 2, pp. 125-128, 2005.

[7] W. J. Rugh and J. S. Shamma, "Research on gain scheduling," Automatica, vol. 36, no. 10, pp. 1401-1425, 2000.

[8] G. Becker, A. Packard, D. Philbrick, and G. Balas, "Control of parametrically-dependent linear systems: a single quadratic Lyapunov approach," in Proceedings of the American Control Conference, pp. 2795-2799, San Francisco, Calif, USA, June 1993.

[9] Y.-Y. Cao, J. Lam, and Y.-X. Sun, "Static output feedback stabilization: an ILMI approach," Automatica, vol. 34, no. 12, pp. 1641-1645, 1998.

[10] L. El Ghaoui and V. Balakrishnan, "Synthesis of fixed-structure controllers via numerical optimization," in Proceedings of the 33rd IEEE Conference on Decision and Control (DC '94), vol. 3, pp. 2678-2683, Lake Buena Vista, Fla, USA, December 1994.

[11] L. El Ghaoui, F. Oustry, and M. Aitrami, "A cone complementarity linearization algorithm for static output-feedback and related problems," IEEE Transactions on Automatic Control, vol. 42, no. 8, pp. 1171-1176, 1997.

[12] K. M. Grigoriadis and R. E. Skelton, "Low-order control design for LMI problems using alternating projection methods," Automatica, vol. 32, no. 8, pp. 1117-1125, 1996.

[13] T. Iwasaki and R. E. Skelton, "The $X Y$-centring algorithm for the dual LMI problem: a new approach to fixed-order control design," International Journal of Control, vol. 62, no. 6, pp. 1257 1272, 1995.

[14] S. Boyd, L. El Ghaoui, E. Feron, and V. Balakrishnan, Linear Matrix Inequalities in System and Control Theory, vol. 15 of SIAM Studies in Applied Mathematics, SIAM, Philadelphia, Pa, USA, 1994.

[15] P. Gahinet and P. Apkarian, "A linear matrix inequality approach to $\mathscr{H}_{\infty}$ control," International Journal of Robust and Nonlinear Control, vol. 4, no. 4, pp. 421-448, 1994.

[16] W. Xie and T. Eisaka, "Design of LPV control systems based on Youla parameterisation," IEE Proceedings of Control Theory and Applications, vol. 151, no. 4, pp. 465-472, 2004.

Wei Xie: College of Automation Science and Technology, South China University of Technology, Guangzhou 510641, China

Email address: weixie@scut.edu.cn 


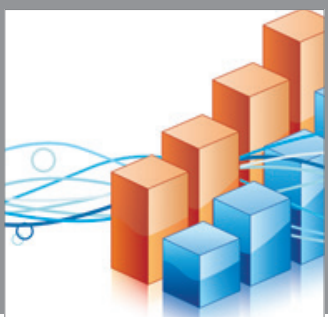

Advances in

Operations Research

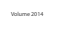

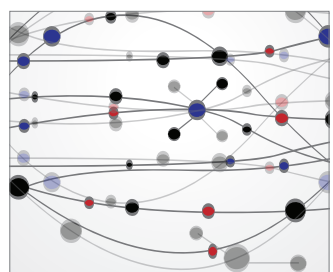

\section{The Scientific} World Journal
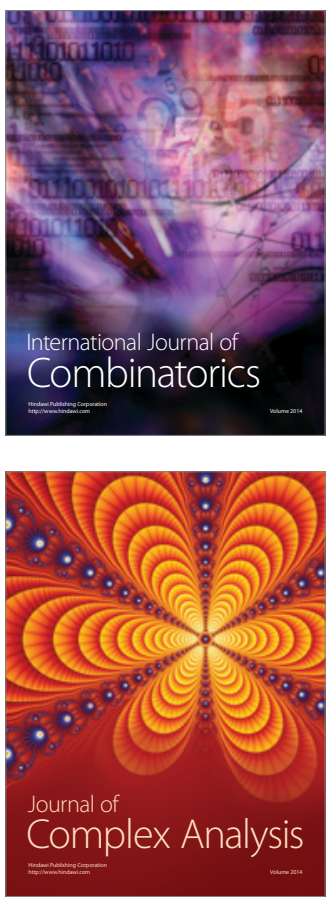

International Journal of

Mathematics and

Mathematical

Sciences
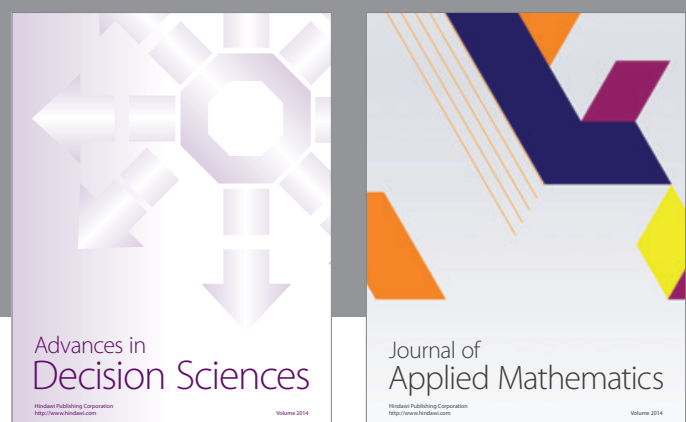

Journal of

Applied Mathematics
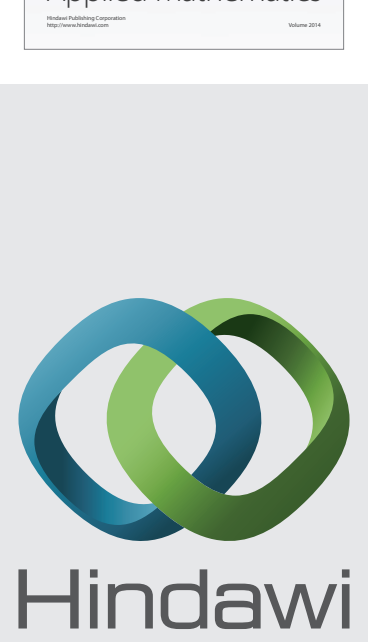

Submit your manuscripts at http://www.hindawi.com
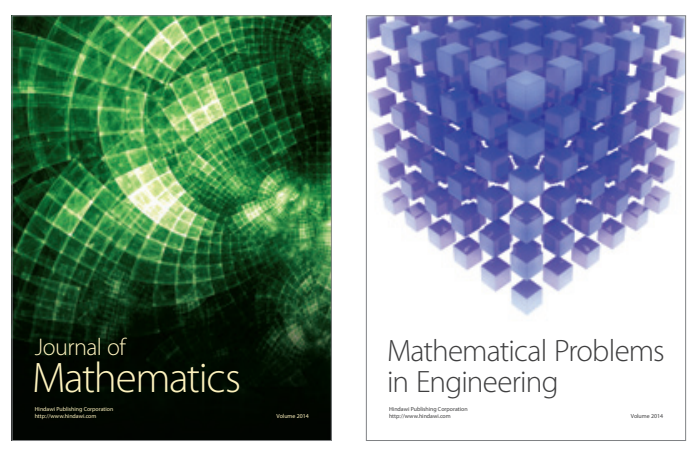

Mathematical Problems in Engineering
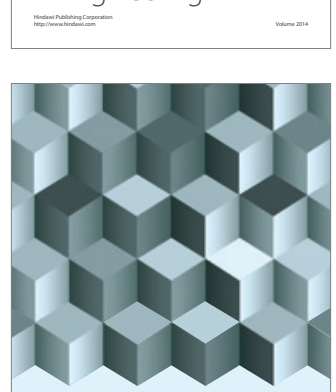

Journal of

Function Spaces
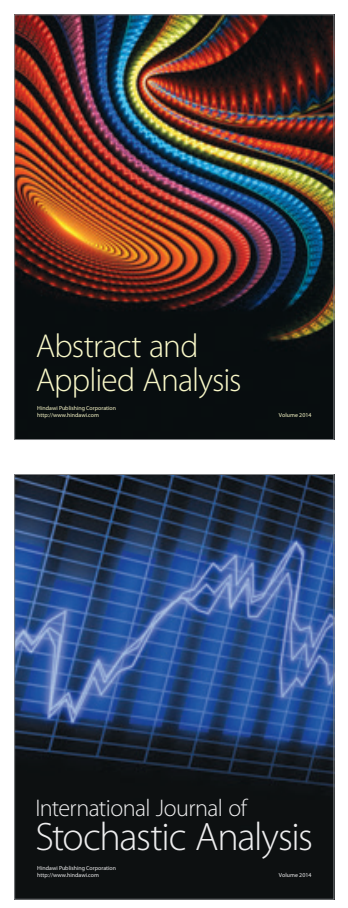

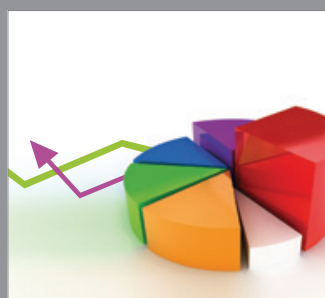

ournal of

Probability and Statistics

Promensencen
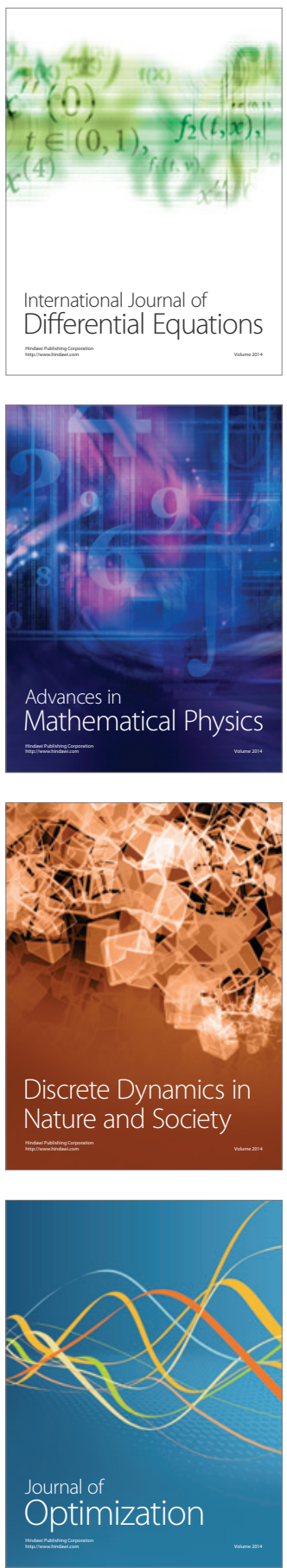